\title{
DECam photometry reveals extra-tidal stars around the Milky Way globular cluster NGC 6864 (M75)
}

\author{
Andrés E. Piatti ${ }^{1,2 \star}$ \\ ${ }^{1}$ Instituto Interdisciplinario de Ciencias Básicas (ICB), CONICET-UNCUYO, Padre J. Contreras 1300, M5502JMA, Mendoza, Argentina \\ ${ }^{2}$ Consejo Nacional de Investigaciones Científicas y Técnicas, Godoy Cruz 2290, C1425FQB, Buenos Aires, Argentina
}

Accepted XXX. Received YYY; in original form ZZZ

\begin{abstract}
Globular clusters are prone to lose stars while moving around the Milky Way. These stars escape the clusters and are distributed throughout extended envelopes or tidal tails. However, such extra-tidal structures are not observed in all globular clusters, and yet there is no structural or dynamical parameters that can predict their presence or absence. NGC6864 is an outer halo globular cluster with reported no observed tidal tails. We used Dark Energy Camera (DECam) photometry reaching $\sim 4$ mag underneath its main sequence turnoff to confidently detect an extra-tidal envelope, and stellar debris spread across the cluster outskirts. These features emerged once robust field star filtering techniques were applied to the fainter end of the observed cluster main sequence. NGC 6864 is associated to the Gaia-Enceladus dwarf galaxy, among others 28 globular clusters. Up-to-date, nearly $64 \%$ of them have been targeted looking for tidal tails and most of them have been confirmed to exhibit tidal tails. Thus, the present outcomes allow us to speculate on the possibility that Gaia-Enceladus globular clusters share a common pattern of mass loss by tidal disruption.
\end{abstract}

Key words: Galaxy: globular clusters: general - techniques: photometric - globular clusters: individual: NGC 6864

\section{INTRODUCTION}

Globular cluster tidal tails have become sensitive tracers of the nature and distribution of dark matter in the Milky Way (Bonaca et al. 2019). There are indications from Nbody simulations that globular clusters might originally have been embedded inside substantial mini-halos of dark matter (Peñarrubia et al. 2017; Boldrini et al. 2020). Nevertheless, there is an ongoing debate as to whether the gaps observed in streams, as for example, along the GD-1 stream and the Pal 5 tidal tails (Grillmair \& Dionatos 2006a,b), are due to dark matter sub-halos, or to epicyclic motions of a continuous stream of stars escaping the clusters as a consequence of globular cluster tidal interaction with the Milky Way (Küpper et al. 2010, 2012). From an observational point of view, there have been many investigations of the outermost regions of globular clusters with the goal of detecting extratidal structures and tidal tails. From a recent comprehensive compilation of the relevant observational results obtained to date of 53 globular clusters, Piatti \& Carballo-Bello (2020) found that 14 globular clusters have observed tidal tails and 17 show no detectable signatures of extra-tidal structures.

* E-mail: andres.piatti@unc.edu.ar
They showed that there is no currently known parameters that enable us to confidently predict the presence or absence of tidal tails for any given globular cluster.

NGC 6864 (M75) is a Milky Way globular cluster located at a Galactocentric distance $R_{G C}$ of $14.7 \mathrm{kpc}$ (Harris 1996, December 2010 Edition). In the context of that a fraction of the Milky Way globular cluster population might have originated in and have been accreted with already extinct dwarf galaxies, Carballo-Bello et al. (2014) searched for the remnants of the progenitor galaxy that might be still populating the surroundings of this cluster. From crosscorrelation and isochrone-fitting methods, they found no evidence of distinct stellar population concentrated at a specific distance within the probed colour-magnitude range. The lack of tidal debris led them to wonder whether they could rule out an accretion origin for this globular cluster. They posed that either the progenitor galaxy was not massive or that the accretion event took place early in the Milky Way hierarchical assembly (Bonaca et al. 2021).

de Boer et al. (2019) took advantage of Gaia DR2 data (Gaia Collaboration et al. 2018) to study the outskirts of NGC 6864, using the Gaia proper motions to select cluster's members. They assumed that cluster's stars distributed in the periphery of the cluster share the mean cluster's proper 
motion and limited their star sample to those with $G<$ 20 mag. This means that they made use of red giant stars that move similarly to the cluster's body. However, since these stars are either in the process of escaping the cluster or orbiting within a dark matter mini-halo, their velocity behavior should be somewhat different from that of stars still bound to the cluster (Starkman et al. 2019), so that proper motions by themselves are not sufficient for reliable membership determination of tidal tails' stars. On the other hand, less massive main sequence stars are prone to leave the cluster more easily than red giant ones. Detected tidal tails in globular clusters are frequently composed by cluster main sequence stars that lie $\sim 1-2$ mag underneath the main sequence turnoff (see, e.g., Jordi \& Grebel 2010; Myeong et al. 2017; Shipp et al. 2018). The two selection criteria applied by de Boer et al. (2019) led them to conclude that the cluster does not exhibit tidal tails, which is an expected result according to the reasons mentioned above.

The ex-situ origin of NGC 6864 was suggested by Massari et al. (2019) from the examination of the space of integrals of motion of Milky Way globular clusters, and confirmed by Forbes (2020), who combined kinematic properties with ages, metallicities, and alpha-elements, that remain largely unchanged over time. It belongs to a sample of 28 globular clusters probably associated to the massive GaiaEnceladus galaxy, that underwent a major merger with the Milky Way 8-11 Gyr ago (Naidu et al. 2021, and references therein). Nearly $64 \%$ of these globular clusters (18 objects) have studies of their outermost regions (Piatti \& CarballoBello 2020), which show that 13 of them (72\%) exhibit tidal tails or extra-tidal features that are different from tidal tails. A complete census of Gaia-Enceladus globular clusters with tidal tails points to the need of studies of the outskirts of the remaining 10 objects, and of the reanalysis of those few ones with no previous detection of tidal tails (NGC 6205, 6229, 6864). We note that Jordi \& Grebel (2010) did not detect any tidal tails in the Gaia-Enceladus globular cluster NGC 6341, which were recently uncovered by Thomas et al. (2020). This example illustrates that the combination of different photometry depths and filtering techniques can produce meaningful distinct results.

In this work we performed a novel search for signatures of tidal tails around NGC 6864. Previous studies indicate the lack of observational evidence of such extra-tidal features, which somehow is counterintuitive for an accreted outer halo globular cluster. In Sections 2 and 3 we describe the data used and the developed method applied to highlight the outer faint cluster stellar structures, respectively. Section 4 deals with the analysis of the resulting stellar density maps.

\section{COLLECTED DATA}

The Dark Energy Camera (DECam), which is attached to the prime focus of the 4-m Blanco telescope at Cerro Tololo Inter-American Observatory (CTIO), was used to image the field of NGC 6864. DECam is an array of 62 identical chips with a scale of $0.263 \operatorname{arcsec}_{\text {pixel }}{ }^{-1}$ that provides a $3 \mathrm{deg}^{2}$ field of view (Flaugher et al. 2015). The images, obtained as part of the observing program CTIO 2019B-1003 (PI : Carballo-Bello), are publicly available and consist of $4 \times 600$ sec $g$ and $4 \times 400$ sec $r$ exposures, respectively. NGC 7089 is the other globular cluster included in the program with unpublished DECam data; it will be the subject of a forthcoming paper. The data set includes nightly observations of 5 SDSS fields at a different airmass, which were used to derive the atmospheric extinction coefficients and the transformations between the instrumental magnitudes and the SDSS ugriz system (Fukugita et al. 1996).

In order to process the program images, the DECam Community Pipeline (Valdes et al. 2014) was used, while the photometry was obtained from the processed images using the DAOPHOT II/ALLSTAR point-spread-function fitting routines (Stetson et al. 1990). From the resulting photometric catalog, we imposed the restriction of $\mid$ sharpness $\mid \leq 0.5$ to avoid the presence of bad pixels, cosmic rays, background galaxies, and unrecognized double stars in our subsequent analysis. and kept positions and standardized $g$ and $r$ magnitudes of stellar objects that surpassed that criterion. The photometry completeness was estimated from artificial star tests, using the DAOPHOT II routines. Briefly, synthetic stars with magnitudes and positions distributed similarly to those of the measured stars in each image were added, and their photometry was carried out applying the same point-spreadfunction fitting routines, as above. Finally, the resulting magnitudes for the synthetic stars were then compared to those used to create such stars. The $50 \%$ completeness level resulted to be $23.4 \mathrm{mag}$ and $23.3 \mathrm{mag}$ for the $g$ and $r$ bands, respectively (see, also, Piatti et al. 2020, 2021).

Figure 1 (left panel) shows the colour-magnitude diagram (CMD) for a circular region around the cluster centre. With the aim of clearly delineate the cluster main sequence, we used stars distributed inside a circle with radius $0.09^{\circ}$, which is $\sim 70 \%$ the cluster tidal radius (see Section 4 ). Thus, we ensured that most of the stars are likely cluster members. As can be seen, the cluster main sequence is visibly thin and spans over nearly 4 mag underneath its main sequence turnoff. NGC 6864 is projected along a line-of-sight that does not show a severe contamination of field stars. The figure (right panel) shows that for an annulus centred on the cluster with an internal radius more than 6 times larger than the cluster tidal radius, and with the same cluster area for comparison purposes, the number of stars along the cluster main sequence is notably low. On purpose, the cluster CMD was built using magnitudes and colours corrected by interstellar reddening, whose median value across the whole field is low. Figure 2 shows the variation of the interstellar reddening $E(B-V)$ across the DECam field of view, with $E(B-V)$ values from Schlafly \& Finkbeiner (2011) as provided by the NASA/IPAC Infrared Science Archive ${ }^{1}$. In order to construct the reddening map, we used a uniform grid of Right Ascension and Declination values covering the entire DECam field of view in steps of 1 arcmin, respectively. We corrected the $g$ and $r$ magnitudes of each star by using the $E(B-V)$ colour excesses according to the positions of the stars in the sky.

1 https://irsa.ipac.caltech.edu/ 


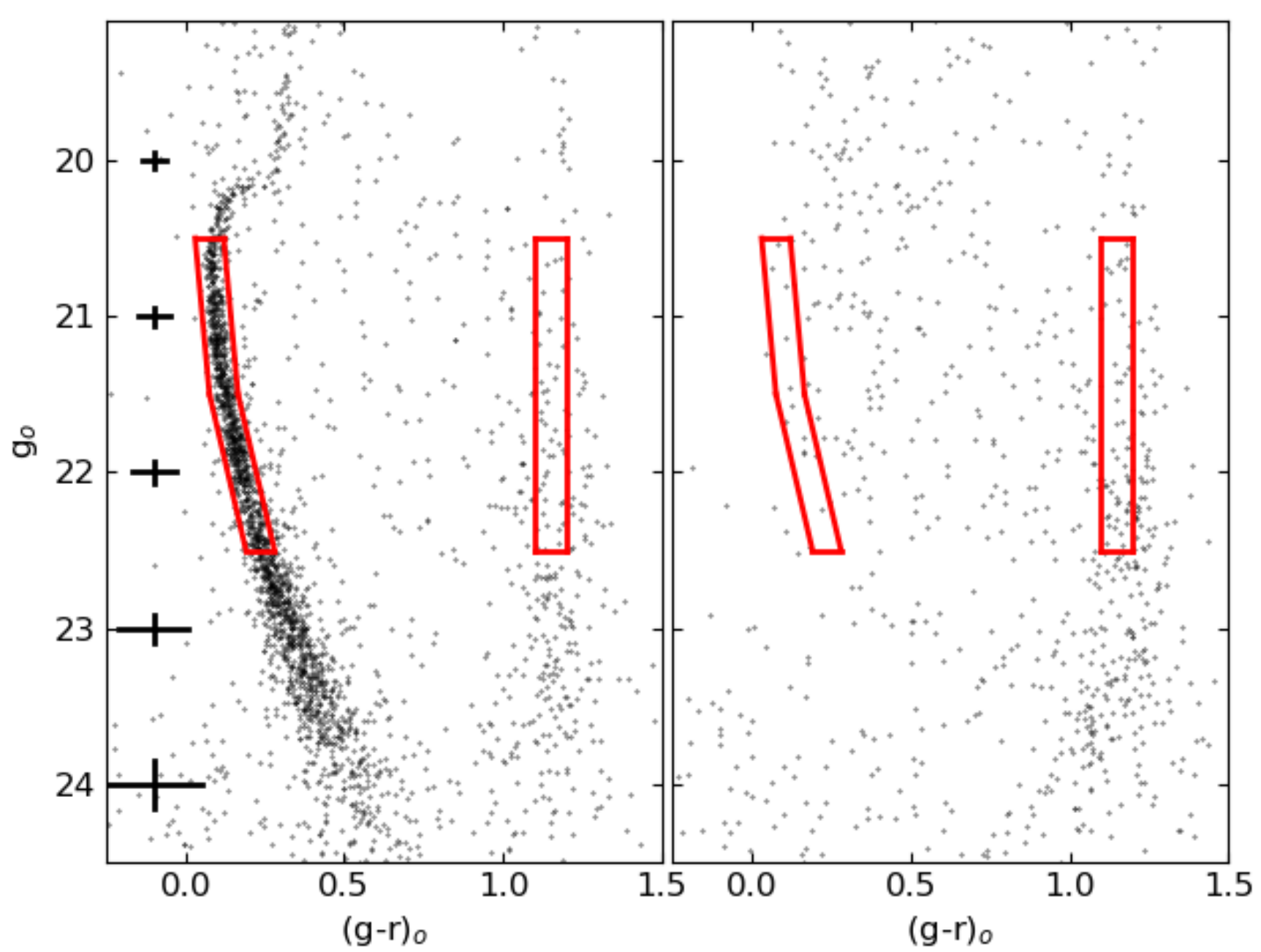

Figure 1. Intrinsic CMDs for a circle of radius $0.09^{\circ}$ centred on the cluster (left panel), compared with that for an annular field region with an equal cluster area and internal radius of $0.8^{\circ}$ (right panel). Segments along the cluster main sequence and the composite field population are drawn, respectively, in red. Typical error bars are also indicated.

\section{STELLAR DENSITY MAPS}

Carballo-Bello et al. (2014) used a template region along the cluster main sequence defined from theoretical isochrones and counted the number of stars inside it, given different weights to the stars according to their distances to the central line of the template region. Thus, they assigned more weight to stars placed along the cluster main sequence ridge line. However, such a criterion does not get rid of field stars, that populate the cluster main sequence too. de Boer et al. (2019) employed red giant stars with proper motions similar to the mean cluster proper motion. We note that mass segregation also makes more massive stars to be more centrally concentrated (Khalisi et al. 2007, and references therein). We here decided to used main sequences stars, because stars with smaller masses can be found more easily far away from the cluster main body (Carballo-Bello et al. 2012). For this reason, most of the studies devoted to searching for extratidal structures have used relatively faint main sequence stars (see, e.g., Olszewski et al. 2009; Saha et al. 2010). We also cleaned the cluster main sequence from field star contamination, so that the distribution in the sky of the stars that remained unsubtracted represents the intrinsic spatial distribution of cluster members.

Figure 1 shows a region on the cluster main sequence enclosed within red boundaries. We selected that portion of the cluster main sequence to build the respective stellar density map, once it was cleaned from field star contamination. The method applied for such a decontamination of field stars was devised by Piatti \& Bica (2012), and was satisfactorily applied elsewhere (e.g., Piatti 2017a, 2018; Piatti et al. 2018, and references therein). The procedure proved to be successful for clusters projected on to crowded fields and affected by differential reddening (see, e.g., Piatti 2017b; Piatti \& Carballo-Bello 2019; Piatti \& Fernández-Trincado 2020 , and references therein). In order to clean the selected cluster main sequence segment, we need to compare it with a reference star field, and then to properly eliminate from the former a number of stars equal to that found in the latter, bearing in mind that the magnitudes and colours of the eliminated stars in the cluster main sequence segment must reproduce the respective magnitude and colour distributions in the reference star field main sequence. For that purpose, 


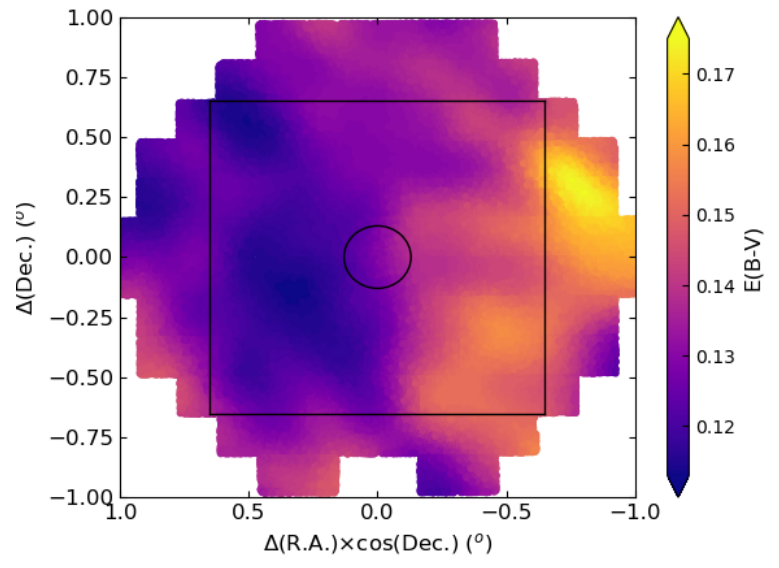

Figure 2. Reddening variation across the field of NGC 6864. The solid box delimites the internal boundaries of the adopted field star reference field. The circle corresponds to the cluster tidal radius (see Section 4).

we traced a square in the DECam field of view that splits the whole covered field in two equal areas; the reference star field region being the outermost one.

The methodology to select stars to subtract from the cluster main sequence segment consists in defining boxes centred on the magnitude and colour of each star of the reference star field; then to superimpose them on the star cluster main sequence segment, and finally to choose one star per box to subtract. We started with boxes with size of $\left(\Delta g_{0}, \Delta(g-r)_{0}\right)=(0.25 \mathrm{mag}, 0.10 \mathrm{mag})$ centred on the $\left(g_{0}\right.$, $\left.(g-r)_{0}\right)$ values of each reference field star, in order to guarantee to find a star in the cluster main sequence segment with the magnitude and colour within the box boundary. In the case that more than one star is located inside the cluster main sequence segment, the closest one to the centre of that (magnitude, colour) box is subtracted. The magnitude and colour errors of the stars in the cluster main sequence segment were taken into account while searching for a star to be subtracted. With that purpose, we allowed the stars in the cluster main sequence segment to vary their magnitudes and colours within an interval of $\pm 1 \sigma$, where $\sigma$ represents the errors in their magnitude and colour, respectively. We allowed up to 1000 random combination of their magnitude and colour errors.

Because of the relatively large extension of the cleaned cluster field $\left(1.3^{\circ} \times 1.3^{\circ}\right.$; see Fig. 2$)$, we imposed the condition that the spatial positions of the stars to be subtracted from the cluster main sequence segment were chosen randomly. Thus, we avoided spurious overdensities in the resulting cluster stellar density map driven by the subtraction of stars from the cluster main sequence segment that are located nearly in the same sky regions. In practice, for each reference field star, we first randomly selected the position of a box of $0.05^{\circ}$ a side in the cluster field (the square area in Fig. 2) where to subtract a star. We then looked for a star with $\left(g_{0},(g-r)_{0}\right)$ values within the (magnitude, colour) box defined as described above, taking into account the photometric errors. If no star is found in the selected spatial box, we repeated the selection a thousand times, otherwise we enlarged the box size in steps of $0.01^{\circ}$ a side, to iterate the process. The outcome of the cleaning procedure is a cluster main sequence segment that likely contains only cluster members; their spatial distributions relies on a random selection. For this reason, we executed 1000 times the decontamination procedure, and defined a membership probability $P(\%)$ as the ratio $N / 10$, where $N$ is the number of times a star was found among the 1000 different outputs. In the subsequence analysis we only kept stars with $P>70 \%$.

The observed stellar density map for stars distributed within the cluster main sequence segment was built using the scikit-learn software machine learning library (Pedregosa et al. 2011) and its kernel density estimator (KDE). We employed a grid of $500 \times 500$ boxes on the cluster field and allowed the bandwidth to vary from $0.005^{\circ}$ up to $0.040^{\circ}$ in steps of $0.005^{\circ}$. The adopted optimal value for the bandwidth turned out to be $0.025^{\circ}$. The background level was estimated from the stars distributed in the reference star field. We split this area in boxes of $0.10^{\circ} \times 0.10^{\circ}$ and counted the number of stars inside them, and randomly shifted the boxes by $0.05^{\circ}$ along the abscisas or ordinates and repeated the star counting, with the aim of enlarging the statistics. Finally, we derived the mean value of the star counts coming from all the defined boxes. We also estimated its standard deviation from a thousand Monte Carlo realizations of the stellar density map, shifting the positions of the stars along $\Delta(\mathrm{RA}) \times \cos (\mathrm{Dec})$ or $\Delta($ Dec.) randomly (one different shift for each star) before recomputing the density map. Figure 3 depicts the resulting observed density map (left panel) that shows the absolute deviation from the mean value in the field in units of the standard deviation, namely: $\eta=$ (signal - mean value)/standard deviation. The cleaned stellar density map was constructed using the same recipe and is shown in the right panel of Fig. 3. As can be seen, there are several scattered stellar debris around the cluster. The direction toward the Milky Way centre and that of the cluster motion vector(Baumgardt et al. 2019) are also drawn with grey and blue arrows, respectively.

For the sake of the reader, Fig. 4 illustrates the distribution of stars in the CMD segment chosen for building the cluster stellar density map (top-left panel); that of the reference star field (top-middle panel), and the resulting cleaned CMD segment (top-right panel). The bottom panels show their respective spatial distributions. Stars in the bottomleft and bottom-right panels were used to build left and right panels of Fig. 3, respectively. As can be seen, the cleaning procedure subtracted stars reproducing the magnitude and colour distributions of stars in the reference star field and randomly spatially distributed, particularly for stars located outside the cluster tidal radius. Fig. 5 shows the $g_{0}$ distributions of stars in the top-left and top-right panels of Fig. 4 represented by red and black lines, respectively. For comparison purposes, the $g_{0}$ distribution of stars in the reference star field is also shown with a blue line. The cleaned cluster CMD segment for stars located outside the cluster radius shows a slight decrease of the number of stars toward fainter magnitudes. Such an effect witnesses the cluster mass loss, being less massive stars prone to leave the cluster more easily, because they are the first to cross the Jacobi radius once they reach the cluster boundary driven by two-body relaxation. 

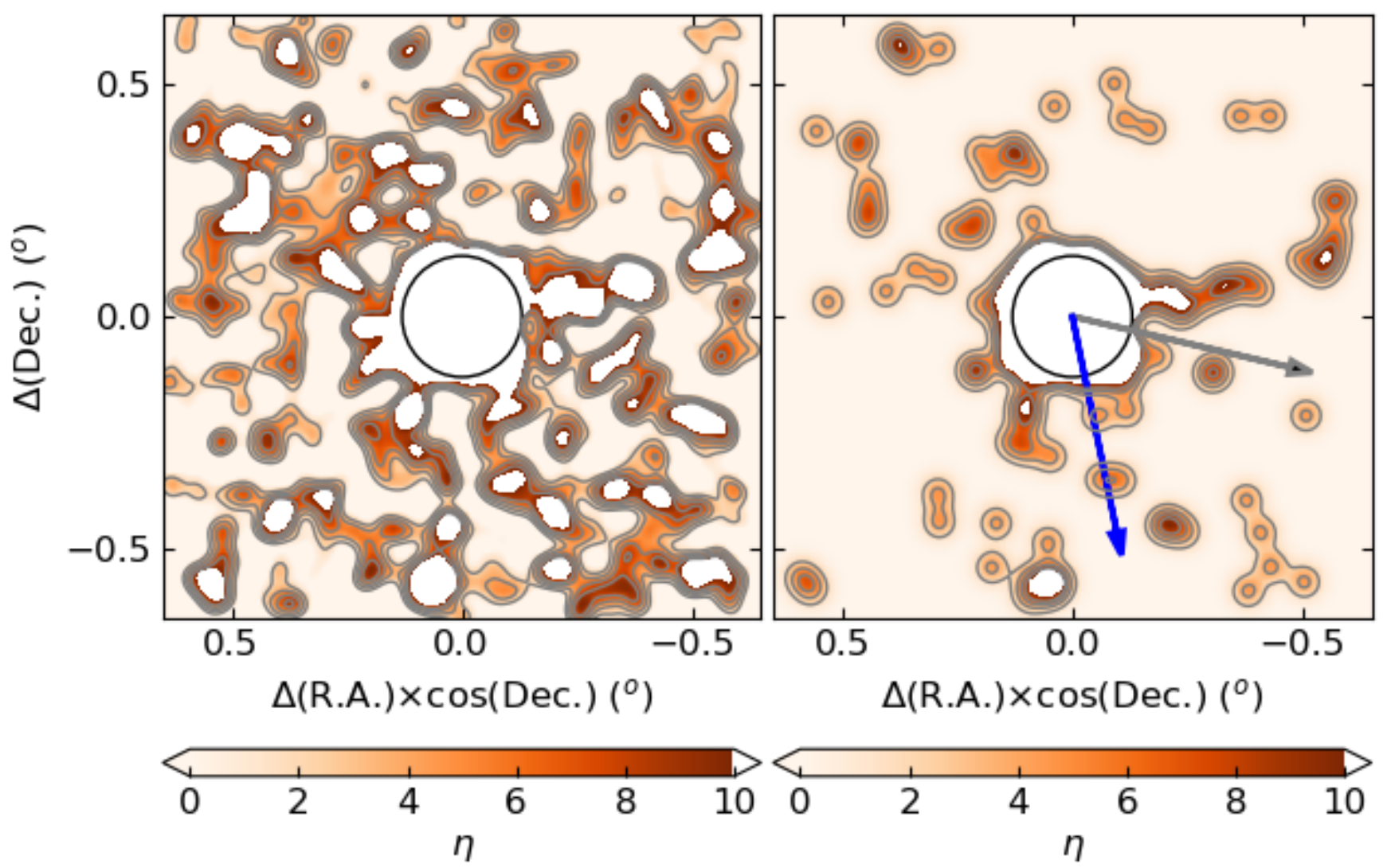

Figure 3. Observed cluster main sequence segment stellar density map (left panel) and that build for stars that remained unsubstracted from the cleaning procedure and with $P>70 \%$ (right panel). The black circle centred on the cluster indicates the cluster tidal radius (see Section 4). Contours for $\eta=2,4,6$, and 8 are also shown. We have painted white stellar densities with $\eta>10$ in order to highlight the least dense structures. The grey and blue arrows in the right panel show the direction to the Milky Way centre and that of the motion of the cluster (Baumgardt et al. 2019), respectively.

\section{ANALYSIS AND DISCUSSION}

Although the cleaned cluster stellar density map was built from stars that have chances higher than $70 \%$ of being genuine cluster members, we additionally validated the field star decontamination procedure by applying it to a sample of stars located in a CMD region where only the presence of field stars is expected. We drew in Fig. 1 a vertical red rectangle spanning the same magnitude range as the cluster main sequence segment and placed toward redder colours, where field stars are numerous. We note that those stars are located throughout the entire DECam field of view, as they are seen in both the cluster and reference star field CMDs (see left and right panels of Fig. 1). Therefore, we cleaned the vertical segment composed by stars distributed across the cluster field from stars that have magnitudes and colours within the boundaries of the vertical segment but are located in the reference star field. Under the assumption that both populations of stars have a common origin (they belong to the composite Milky Way field population), the cleaned stellar density map should show no resulting stellar overdensities.

We illustrate in Fig. 6 the observed stellar density map built for the vertical segment (left panel), where stars seem not to be uniformly distributed. For example, there are more stars spread in the southwest quadrant than toward the north from the cluster centre. We note that vertical segment stars also populate the circle drawn with a radius equal to the cluster tidal radius and centred on the cluster. The resulting field star decontaminated stellar density map (right panel of Fig. 6) shows that there are nearly no residuals with a membership probability $P>70 \%$, except an small overdensity located in the south-west quadrant with $\eta<2$. This stellar excess does not coincide with any extra-tidal features uncovered in Fig. 3 (right panel). Therefore, we concluded that the uncovered cluster main sequence segment stellar debris are intrinsic structural extra-tidal features of NGC 6864. These findings show that the combination of appropriate data sets (in this case relatively deep cluster photometry) 


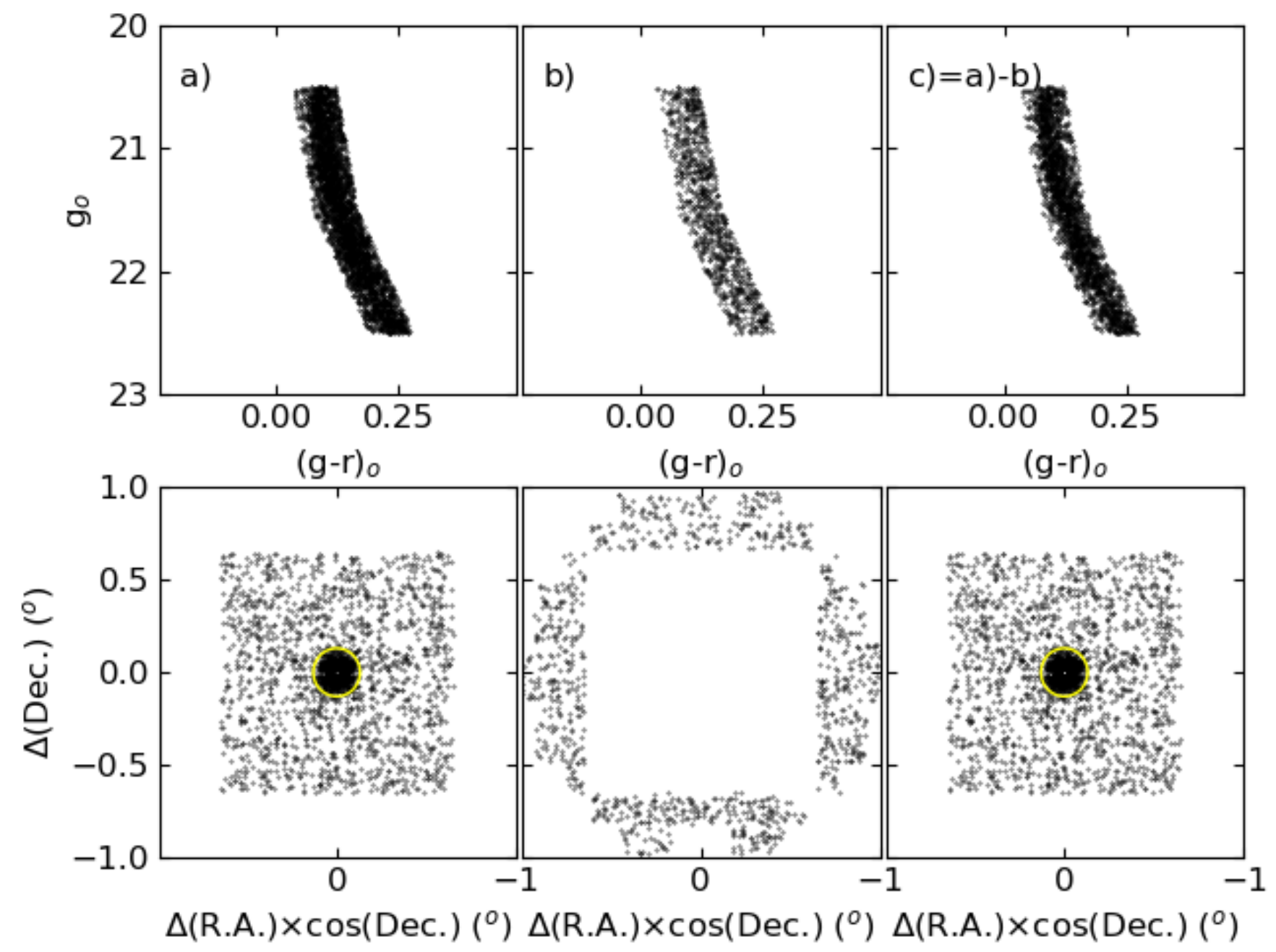

Figure 4. Top panels: CMD of individual stars used in the CMD cleaning procedure (see Section 3 for details), namely: a) all the stars in the cleaned area; b) all the stars in the reference field, and c) stars that remained unsubtracted with $P>70 \%$. Bottom panels: Spatial distributions of the stars in the CMDs built above them, respectively. The yellow circles correspond to the cluster tidal radius (see Section $4)$.

and robust field star filtering techniques are suitable to uncover extra-tidal structures in globular clusters. The stellar debris detected around NGC 6864 make it to be added to the number of outer halo globular clusters with observed extra-tidal features associated to the accreted massive GaiaEnceladus dwarf galaxy. This finding confirms that most, if not all, of the globular clusters associated to Gaia-Enceladus exhibit extra-tidal stellar ovendensities (Carballo-Bello et al. 2014; Piatti \& Carballo-Bello 2020; Bonaca et al. 2021). Nevertheless, such an speculative possibility will be comprehensively answered once the complete sample of Gaia-Enceladus clusters is targeted by studies that search for those features around them.

NGC 6864 shows an excess of stars that extends beyond the cluster tidal radius. Harris (1996) compiled a tidal radius of $0.09^{\circ}$, while Carballo-Bello et al. (2012) estimated a value of $0.11^{\circ}$. de Boer et al. (2019) fitted King (1966) and Wilson (1975) models, a lowered isothermal model explorer in PYTHON (LIMEPY; Gieles \& Zocchi 2015), and a spherical potential escapers stitched model (SPES, Clay- don et al. 2019) to the number density profile constructed from cluster red giants. They concluded that both King and Wilson models are too simplistic, and LIMEPY or SPES models are needed to explain the distribution of stars simultaneously in the inner and outer regions. While inspecting their results (see their figure A13), we found that the best solution is that of the SPES model, whose resulting tidal radius is $0.13^{\circ}$. We would like to note that the different tidal radii derived above, and hence the name of extra-tidal stars given to those stars located beyond that distance from the custer centre, are different from the Jacobi radius estimated by Balbinot \& Gieles $\left(2018,0.46^{\circ}\right)$. According to de Boer et al. (2019), their SPES tidal radii are similar in general to the Jacobi radii for Milky Way globular clusters, although their Figure 10 reveals some noticeable differences.

We built the cluster stellar radial profile using all the stars distributed in the stellar density map of Fig. 3. We focused on the outermost region $\left(r>0.09^{\circ}\right)$ where radial variations of the photometry completeness are negligible and where we are interested in finding cluster extra-tidal 


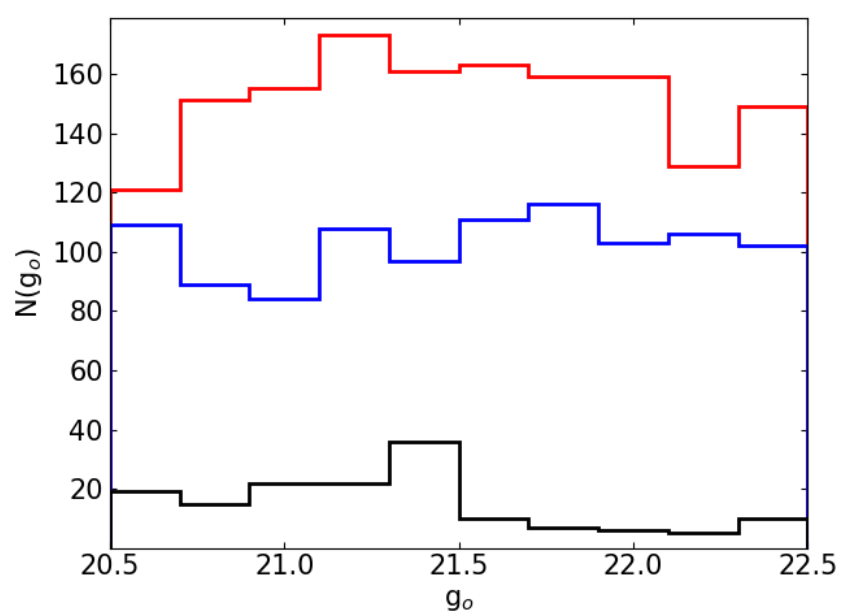

Figure 5. $g_{0}$ distributions in panels a) and c) of Fig. 4 for stars located outside the cluster tidal radius, drawn with red and black lines, respective. For comparison purpose, the $g_{0}$ distribution of reference field stars is also shown with a blue line.

features. In order to generate the stellar radial profile, we counted the number of stars in annuli of $0.025^{\circ}$ wide. Figure 7 depicts the resulting observed radial profile represented with open circles. We then estimated the mean background level using all the points located at distances larger than $0.65^{\circ}$ from the cluster centre, which turned out to be $\log \left(N_{b g} / N_{0}\right)=-1.90 \pm 0.06$. Once the mean background level was subtracted from the observed radial profile, we obtained the radial profile represented by filled circles in Fig. 7. For comparison purposes we superimposed the field star cleaned radial profile constructed from counts of stars found in the decontaminated cluster CMD with $P>70 \%$ (open trialgles). We fitted the normalized field star cleaned radial profile with a King (1962)'s model using the known cluster core radius $\left(0.0015^{\circ}\right.$; Harris 1996; Baumgardt et al. 2019). The derived tidal radius turned out to be $(0.20 \pm 0.04)^{\circ}$. As can be seen in Fig. 7, there is an excess of stars beyond the fitted King (1962) profile that follows a power law $\propto r^{-\alpha}$, with slope $\alpha$ equals to 3.25 . This value is in between those found in Milky Way globular clusters with extended halolike structures, e.g., NGC 1851 and 47 Tuc (Olszewski et al. 2009; Piatti 2017b, $\alpha=1.24$ ) and the abrupt fall of the $r^{-4}$ law suggested by Peñarrubia et al. (2017) as a prediction of expected stellar envelopes of Milky Way globular clusters embedded in dark mini-haloes.

In general, globular clusters whose orbits are relatively more eccentric and very inclined with respect to the Milky Way plane, have undergone a larger amount of mass loss by tidal disruption (see, Fig. 1 of Piatti \& Carballo-Bello 2020). The inclination of the orbit and eccentricity of NGC 6864 are $i=49.76^{\circ}$ and $\epsilon=0.79$, respectively (Piatti 2019). However, the cluster has lost $16 \%$ of its initial mass from tidal disruption, which is a relative low value as compared with the values for other globular clusters with similar inclinations of their orbits and eccentricities (> 30\%; Piatti et al. 2019). NGC 6864 is at $14.7 \mathrm{kpc}$ from the Galactic centre and its peri- and apogalactic distances are 2.1 and $18.00 \mathrm{kpc}$, respectively (Baumgardt et al. 2019). Because of it varying galactocentric distance, the cluster's size changes, reaching the biggest extension at its apogalactic distance. For this reason, extra-tidal features could be embraced by the dynamically enlarged cluster volume and therefore could not be observed. This does not seem to be the case of NGC 6864. Likewise, perturbations along the globular clusters' tidal tails such as density variations and changes in their directions have been observed (Bonaca et al. 2019, 2020). Unfortunately, the DECam field of view is not large enough as to detect tidal tails.

The observed stellar excesses around NGC 6864 (within a field of $1.3^{\circ} \times 1.3^{\circ}$ ) and those found in most of the globular clusters associated to Gaia-Enceladus with studies of their outermost regions can be interpreted to the light of the theory of kinematically chaotic and non-chaotic systems (see, e.g. Price-Whelan et al. 2016a,b; Pérez-Villegas et al. 2018). We note that not every Milky Way globular cluster presents tidal tails and that Piatti \& Carballo-Bello (2020) using a comprehensive compilation of globular clusters, did not find any clues as to understand why tidal tails are not seen in every globular clusters. They explored different structural and kinematical properties and found that globular clusters behave similarly, independently of the presence of tidal tails or any other kind of extra-tidal feature, or the absence thereof. Recently, Mestre et al. (2020) compared the behavior of simulated streams embedded in chaotic and non-chaotic regions of the phase-space. They find that typical gravitational potentials of host galaxies can sustain chaotic orbits, which in turn do reduce the time interval during which streams can be detected. Therefore, tidal tails in some globular clusters are washed out afterwards they are generated to the point at which it is imposible to detect them. This is the case of innermost globular clusters, with apogalactic distances smaller than $\sim 6 \mathrm{kpc}$ (Piatti \& Carballo-Bello 2020). Conversely, outer halo globular clusters spend most of their lifetimes moving along non-chaotic orbits, so that their tidal tails remain for a longer period before they are eventually wipe out.

Some recent results suggest that the absence of tidal tails in some globular clusters is related to the fact that they were born within dark matter sub-halos, and role reversal (Starkman et al. 2019; Boldrini \& Vitral 2021; Wan et al. 2021). According to Boldrini et al. (2020) halo globular clusters formed at or near the centre of small dark matter halos and at present day still retain an excess of dark matter above the galactic background dark matter. However, NGC 6205 and NGC 7099, two Gaia-Enceladus globular clusters, do not have observed tidal tails (Jordi \& Grebel 2010; Piatti et al. 2020) and only NGC 6205 shows some evidence of being embedded in a dark matter sub-halo. Furthermore, we matched 25 globular clusters, among them NGC 6205, 7099 and other 8 Gaia-Enceladus globular clusters, tested by Carlberg \& Grillmair (2021) while searching for signatures of being contained within dark matter sub-halos, with the comprehensive compilation of detected tidal tails (see Table 1 of Piatti \& Carballo-Bello 2020) and found that several globular clusters without tidal tails do not show a rising velocity dispersion profiles as expected for those embedded in dark matter sub-halos. Therefore, we cannot rule out considering non-chaotic or chaotic orbits, neither globular clusters formed in dark matter sub-halos to interpret the existence of tidal tails around globular clusters. 

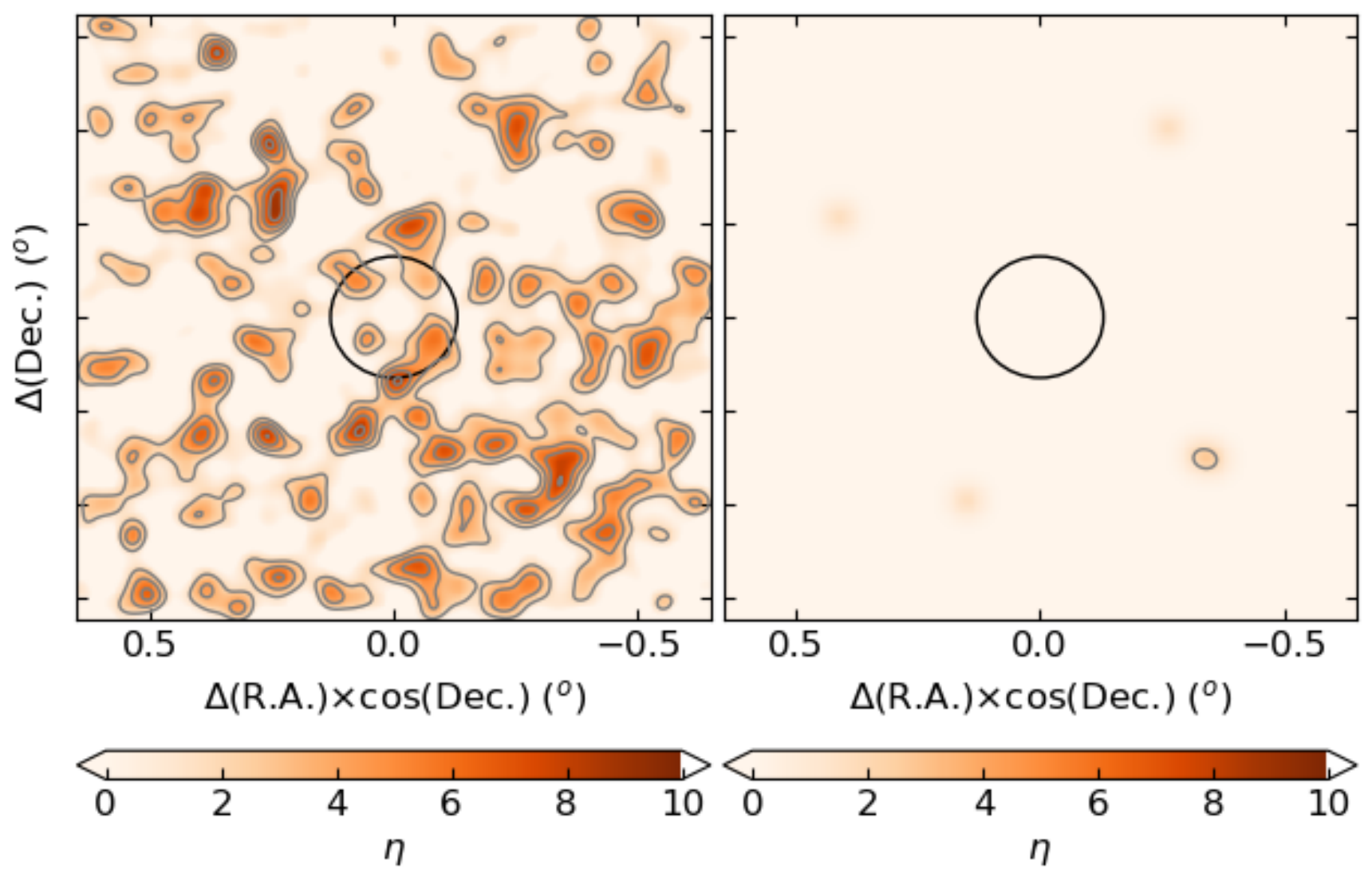

Figure 6. Same as Fig. 3 for the control field segment.

\section{DATA AVAILABILITY}

DECam images used in this work are publicly available at the https://astroarchive.noao.edu/portal/search/\#/searchform webpage.

\section{ACKNOWLEDGEMENTS}

I thank the referee for the thorough reading of the manuscript and timely suggestions to improve it. I also thank the contribution of J.A. Carballo-Bello to an earlier stage of this project.

Based on observations at Cerro Tololo Inter-American Observatory, NSF's NOIRLab (Prop. ID 2019B-1003; PI: Carballo-Bello), which is managed by the Association of Universities for Research in Astronomy (AURA) under a cooperative agreement with the National Science Foundation.

This project used data obtained with the Dark Energy Camera (DECam), which was constructed by the Dark Energy Survey (DES) collaboration. Funding for the DES Projects has been provided by the US Department of Energy, the US National Science Foundation, the Ministry of
Science and Education of Spain, the Science and Technology Facilities Council of the United Kingdom, the Higher Education Funding Council for England, the National centre for Supercomputing Applications at the University of Illinois at Urbana-Champaign, the Kavli Institute for Cosmological Physics at the University of Chicago, centre for Cosmology and Astro-Particle Physics at the Ohio State University, the Mitchell Institute for Fundamental Physics and Astronomy at Texas A\&M University, Financiadora de Estudos e Projetos, Fundação Carlos Chagas Filho de Amparo à Pesquisa do Estado do Rio de Janeiro, Conselho Nacional de Desenvolvimento Científico e Tecnológico and the Ministério da Ciência, Tecnologia e Inovação, the Deutsche Forschungsgemeinschaft and the Collaborating Institutions in the Dark Energy Survey. The Collaborating Institutions are Argonne National Laboratory, the University of California at Santa Cruz, the University of Cambridge, Centro de Investigaciones Enérgeticas, Medioambientales y Tecnológicas-Madrid, the University of Chicago, University College London, the DES-Brazil Consortium, the University of Edinburgh, the Eidgenössische Technische Hochschule (ETH) Zürich, Fermi National Accelerator Laboratory, the University of Illinois at Urbana-Champaign, the 


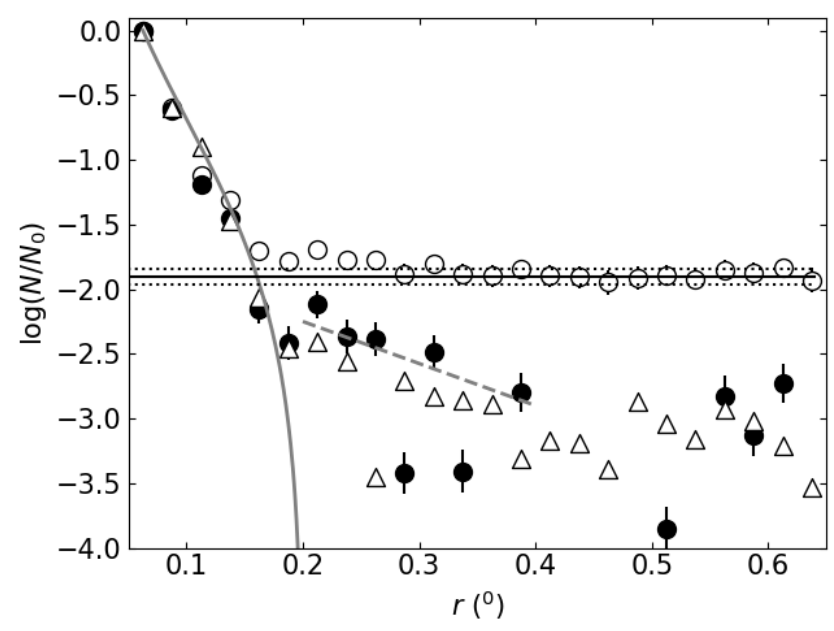

Figure 7. Normalized observed (open circle), mean background subtracted (filled circle) stellar radial profiles and that for stars with $P>70$ (filled circle). The solid and dotted horizontal lines represent the mean background level and its dispersion, respectively. The grey solid line represents the best-fitted King (1962)'s profile, while the dashed line corresponds to a power law with $\alpha=$ 3.25 .

Institut de Ciències de l'Espai (IEEC/CSIC), the Institut de Física d'Altes Energies, Lawrence Berkeley National Laboratory, the Ludwig-Maximilians Universität München and the associated Excellence Cluster Universe, the University of Michigan, NSF's NOIRLab, the University of Nottingham, the Ohio State University, the OzDES Membership Consortium, the University of Pennsylvania, the University of Portsmouth, SLAC National Accelerator Laboratory, Stanford University, the University of Sussex, and Texas A\&M University.

\section{REFERENCES}

Balbinot E., Gieles M., 2018, MNRAS, 474, 2479

Baumgardt H., Hilker M., Sollima A., Bellini A., 2019, MNRAS, 482, 5138

Boldrini P., Vitral E., 2021, arXiv e-prints, p. arXiv:2104.03635

Boldrini P., Mohayaee R., Silk J., 2020, MNRAS, 492, 3169

Bonaca A., Hogg D. W., Price-Whelan A. M., Conroy C., 2019, ApJ, 880, 38

Bonaca A., et al., 2020, ApJ, 889, 70

Bonaca A., et al., 2021, ApJ, 909, L26

Carballo-Bello J. A., Gieles M., Sollima A., Koposov S., MartínezDelgado D., Peñarrubia J., 2012, MNRAS, 419, 14

Carballo-Bello J. A., Sollima A., Martínez-Delgado D., Pila-Díez B., Leaman R., Fliri J., Muñoz R. R., Corral-Santana J. M., 2014, MNRAS, 445, 2971

Carlberg R. G., Grillmair C. J., 2021, arXiv e-prints, p. arXiv:2106.00751

Claydon I., Gieles M., Varri A. L., Heggie D. C., Zocchi A., 2019, MNRAS, 487, 147

Flaugher B., et al., 2015, AJ, 150, 150

Forbes D. A., 2020, MNRAS, 493, 847

Fukugita M., Ichikawa T., Gunn J. E., Doi M., Shimasaku K., Schneider D. P., 1996, AJ, 111, 1748

Gaia Collaboration et al., 2018, A\&A, 616, A1

Gieles M., Zocchi A., 2015, MNRAS, 454, 576
Grillmair C. J., Dionatos O., 2006a, ApJ, 641, L37

Grillmair C. J., Dionatos O., 2006b, ApJ, 643, L17

Harris W. E., 1996, AJ, 112, 1487

Jordi K., Grebel E. K., 2010, A\&A, 522, A71

Khalisi E., Amaro-Seoane P., Spurzem R., 2007, MNRAS, 374, 703

King I., 1962, AJ, 67, 471

King I. R., 1966, AJ, 71, 276

Küpper A. H. W., Kroupa P., Baumgardt H., Heggie D. C., 2010, MNRAS, 401, 105

Küpper A. H. W., Lane R. R., Heggie D. C., 2012, MNRAS, 420, 2700

Massari D., Koppelman H. H., Helmi A., 2019, A\&A, 630, L4

Mestre M., Llinares C., Carpintero D. D., 2020, MNRAS, 492, 4398

Myeong G. C., Jerjen H., Mackey D., Da Costa G. S., 2017, ApJ, 840, L25

Naidu R. P., et al., 2021, arXiv e-prints, p. arXiv:2103.03251

Olszewski E. W., Saha A., Knezek P., Subramaniam A., de Boer T., Seitzer P., 2009, AJ, 138, 1570

Peñarrubia J., Varri A. L., Breen P. G., Ferguson A. M. N., Sánchez-Janssen R., 2017, MNRAS, 471, L31

Pedregosa F., et al., 2011, Journal of Machine Learning Research, 12,2825

Pérez-Villegas A., Rossi L., Ortolani S., Casotto S., Barbuy B., Bica E., 2018, Publ. Astron. Soc. Australia, 35, e021

Piatti A. E., 2017a, ApJ, 834, L14

Piatti A. E., 2017b, ApJ, 846, L10

Piatti A. E., 2018, MNRAS, 477, 2164

Piatti A. E., 2019, ApJ, 882, 98

Piatti A. E., Bica E., 2012, MNRAS, 425, 3085

Piatti A. E., Carballo-Bello J. A., 2019, MNRAS, 485, 1029

Piatti A. E., Carballo-Bello J. A., 2020, A\&A, 637, L2

Piatti A. E., Fernández-Trincado J. G., 2020, A\&A, 635, A93

Piatti A. E., Cole A. A., Emptage B., 2018, MNRAS, 473, 105

Piatti A. E., Webb J. J., Carlberg R. G., 2019, MNRAS, 489, 4367

Piatti A. E., Carballo-Bello J. A., Mora M. D., Cenzano C., Navarrete C., Catelan M., 2020, A\&A, 643, A15

Piatti A. E., Mestre M. F., Carballo-Bello J. A., Carpintero D. D., Navarrete C., Mora M. D., Cenzano C., 2021, A\&A, 646, A176

Price-Whelan A. M., Johnston K. V., Valluri M., Pearson S., Küpper A. H. W., Hogg D. W., 2016a, MNRAS, 455, 1079

Price-Whelan A. M., Sesar B., Johnston K. V., Rix H.-W., 2016b, ApJ, 824, 104

Saha A., et al., 2010, AJ, 140, 1719

Schlafly E. F., Finkbeiner D. P., 2011, ApJ, 737, 103

Shipp N., et al., 2018, ApJ, 862, 114

Starkman N., Bovy J., Webb J., 2019, arXiv e-prints, p. arXiv:1909.03048

Stetson P. B., Davis L. E., Crabtree D. R., 1990, in Jacoby G. H., ed., Astronomical Society of the Pacific Conference Series Vol. 8, CCDs in astronomy. pp 289-304

Thomas G. F., et al., 2020, ApJ, 902, 89

Valdes F., Gruendl R., DES Project 2014, in Manset N., Forshay P., eds, Astronomical Society of the Pacific Conference Series Vol. 485, Astronomical Data Analysis Software and Systems XXIII. p. 379

Wan Z., et al., 2021, MNRAS, 502, 4513

Wilson C. P., 1975, AJ, 80, 175

de Boer T. J. L., Gieles M., Balbinot E., Hénault-Brunet V., Sollima A., Watkins L. L., Claydon I., 2019, MNRAS, 485, 4906

This paper has been typeset from a $\mathrm{T}_{\mathrm{E}} \mathrm{X} / \mathrm{LAT} \mathrm{E}$ X file prepared by the author. 